\title{
Computation of fixed point index and applications to superlinear periodic problem
}

Feng Wang ${ }^{1,2^{*}}$ and Shengjun $\mathrm{Li}^{3}$

"Correspondence:

fengwang188@163.com

'School of Mathematics and Physics, Changzhou University,

Changzhou, 213164, China ${ }^{2}$ College of Science, Hohai

University, Nanjing, 210098, China

Full list of author information is

available at the end of the article

\begin{abstract}
In this paper we compute the fixed point index for A-proper semilinear operators under certain boundary conditions. The proof is based on the partial order method combined with the properties of the fixed point index. As an application, we use the abstract results presented above to study the existence conditions of positive solutions for superlinear first-order and second-order periodic problems.
\end{abstract}

MSC: 34B10; 34B15

Keywords: computation; fixed point index; partial order method; superlinear periodic problem

\section{Introduction}

The topological degree and fixed point index are important theories in nonlinear functional analysis as they have had significant applications as regards obtaining results on the existence and the number of solutions for differential equations [1], differential inclusions [2] and dynamical systems [3, 4]. In recent years, many authors have focused on the computation of the topological degree and fixed point index (see [5-12] and the references therein). It is noticed that the results cited above apply to an operator equation of the form $x=A x$, which is closely connected to fixed point theory. However, in this paper we will be mainly interested in studying a more general operator equation of the form

$$
L x=N x,
$$

where $L$ is not necessarily invertible. During the last three decades, the existence problem for (1.1) has been an interesting topic and has attracted the attention of many researchers $[1,13-16]$ because it has especially broad applications in the existence of periodic solutions of nonlinear differential equations.

Based on the approach of Fitzpatrick and Petryshyn [17], the concept of a fixed point index for A-proper maps related to (1.1) has been introduced by Cremins [18,19]. Since then, some existence results for the semilinear equation (1.1) in cones have been established in $[20,21]$ using the properties of the fixed point index and partial order method. Recently, the computation of the fixed point index for A-proper semilinear operators was proved in [22] under the sublinear case, but to the best of the knowledge of the authors nothing has been published concerning the computation results available in the literature for the

(C) 2015 Wang and Li. This article is distributed under the terms of the Creative Commons Attribution 4.0 International License (http://creativecommons.org/licenses/by/4.0/), which permits unrestricted use, distribution, and reproduction in any medium, provided you give appropriate credit to the original author(s) and the source, provide a link to the Creative Commons license, and indicate if changes were made. 
superlinear case. In this paper, we will continue this study and focus on the computation of the fixed point index with certain boundary conditions in the superlinear case.

The remaining part of the paper is organized as follows. Some preliminaries and a number of lemmas useful to the derivation of the main results are given in Section 2. In Section 3 , we obtain some sufficient conditions that the fixed point index equals $\{1\}$ or $\{0\}$, values more easily applicable. As an application, in Section 4, we use the new results presented in Section 3 to study the existence conditions of positive solutions for first-order and second-order superlinear periodic boundary value problems.

\section{Preliminaries}

In this section, we recall some standard facts on $A$-proper mappings and Fredholm operators.

Let $X$ and $Y$ be Banach spaces with the zero element $\theta, D$ a linear subspace of $X$, $\left\{X_{n}\right\} \subset D$, and $\left\{Y_{n}\right\} \subset Y$ sequences of oriented finite dimensional subspaces such that $Q_{n} y \rightarrow y$ in $Y$ for every $y$ and $\operatorname{dist}\left(x, X_{n}\right) \rightarrow 0$ for every $x \in D$ where $Q_{n}: Y \rightarrow Y_{n}$ and $P_{n}: X \rightarrow X_{n}$ are sequences of continuous linear projections. The projection scheme $\Gamma=\left\{X_{n}, Y_{n}, P_{n}, Q_{n}\right\}$ is then said to be admissible for maps from $D \subset X$ to $Y$. A map $T: D \subset$ $X \rightarrow Y$ is called approximation-proper (abbreviated A-proper) at a point $y \in Y$ with respect to $\Gamma$ if $\left.T_{n} \equiv Q_{n} T\right|_{D \cap X_{n}}$ is continuous for each $n \in \mathbb{N}$ and whenever $\left\{x_{n_{j}} \mid x_{n_{j}} \in D \cap X_{n_{j}}\right\}$ is bounded with $T_{n_{j}} x_{n_{j}} \rightarrow y$, then there exists a subsequence $\left\{x_{n_{j_{k}}}\right\}$ such that $x_{n_{j_{k}}} \rightarrow x \in D$, and $T x=y . T$ is said to be A-proper on a set $D$ if it is A-proper at all points of $Y$.

$L: \operatorname{dom} L \subset X \rightarrow Y$ is a Fredholm operator of index zero if $\operatorname{Im} L$ is closed and $\operatorname{dim} \operatorname{Ker} L=$ codim $\operatorname{Im} L<\infty$. Then $X$ and $Y$ may be expressed as direct sums $X=X_{0} \oplus X_{1}, Y=Y_{0} \oplus Y_{1}$ with continuous linear projections $P: X \rightarrow \operatorname{Ker} L=X_{0}$ and $Q: Y \rightarrow Y_{0}$. The restriction of $L$ to $\operatorname{dom} L \cap X_{1}$, denoted $L_{1}$, is a bijection onto $\operatorname{Im} L=Y_{1}$ with continuous inverse $L_{1}^{-1}$ : $Y_{1} \rightarrow \operatorname{dom} L \cap X_{1}$. Since $X_{0}$ and $Y_{0}$ have the same finite dimension, there exists a continuous bijection $J: Y_{0} \rightarrow X_{0}$.

Cremins $[18,19]$ defined a fixed point index $\operatorname{ind}_{K}([L, N], \Omega)$ for A-proper maps acting on cones, which has the usual properties of the classical fixed point index, that is, existence, normalization, additivity, and homotopy invariance. Let $K$ be a cone in the Banach space $X$, then $X$ becomes a partial ordered Banach space under the partial ordering $\leq$ which is induced by $K . K$ is said to be normal if there exists a positive constant $\tilde{N}$ such that $\theta \leq x \leq y$ implies $\|x\| \leq \tilde{N}\|y\|$. For the concepts and the properties as regards the cone we refer to [23-25]. Here we remark that the results in $[18,19,22]$ hold in partial ordered Banach spaces. Let $\Omega \subset X$ be open and bounded such that $\Omega_{K}=\Omega \cap K \neq \emptyset$. If we let $K_{1}=\left(L+J^{-1} P\right)(K \cap \operatorname{dom} L)$, then $K_{1}$ is a cone in $Y$ and the linear operator $L+J^{-1} P$ is inversely positive by using [18], Proposition 1 .

Throughout this paper we assume that the following conditions are satisfied:

$\left(\mathrm{A}_{1}\right) L: \operatorname{dom} L \rightarrow Y$ is Fredholm of index zero.

$\left(\mathrm{A}_{2}\right) L-\lambda N$ is A-proper for $\lambda \in[0,1]$.

$\left(\mathrm{A}_{3}\right) N$ is bounded and $P+J Q N+L_{1}^{-1}(I-Q) N$ maps $K$ to $K$.

Lemma 2.1 ([26], Lemma 2(a)) If $L_{1}^{-1}$ is compact, then $L-\lambda N$ is A-proper for each $\lambda \in[0,1]$.

To obtain some new methods of computing the fixed point index for the A-proper semilinear operator (1.1), we need the following two lemmas. 
Lemma 2.2 [19] Let $\theta \in \Omega \subset X$. If $L x \neq \mu N x-(1-\mu) J^{-1} P x$ for all $x \in \partial \Omega_{K}$ and $\mu \in[0,1]$, then

$$
\operatorname{ind}_{K}([L, N], \Omega)=\{1\}
$$

Lemma 2.3 [22] If there exists $e \in K_{1} \backslash\{\theta\}$ such that

$$
L x-N x \neq \mu e,
$$

for all $x \in \partial \Omega_{K}$ and all $\mu \geq 0$, then

$$
\operatorname{ind}_{K}([L, N], \Omega)=\{0\}
$$

\section{Main results}

Theorem 3.1 Let $\theta \in \Omega \subset X$ and $L x \neq N x$ for all $x \in \partial \Omega_{K}$. Assume that the following hypotheses hold:

(i) there exists a positive bounded linear operator $B: X \rightarrow X$, such that

$$
\left(N+J^{-1} P\right) x \leq\left(L+J^{-1} P\right) B x, \quad \text { for all } x \in \partial \Omega_{K},
$$

where this partial order is induced by the cone $K_{1}$ in $Y$.

(ii) $r(B) \leq 1$, where $r(B)$ is the spectral radius of $B$.

Then the fixed point index

$$
\operatorname{ind}_{K}\left([L, N], \Omega_{K}\right)=\{1\}
$$

Proof We show that

$$
L x \neq \mu N x-(1-\mu) J^{-1} P x, \quad \forall x \in \partial \Omega_{K}, \mu \in[0,1] .
$$

Suppose the assertion of (3.1) is false. Then there exist $x_{1} \in \partial \Omega_{K}$ and $\mu_{1} \in[0,1]$ such that $L x_{1}=\mu_{1} N x_{1}-\left(1-\mu_{1}\right) J^{-1} P x_{1}$. Since $L x \neq N x \forall x \in \partial \Omega_{K}$, we see that $\mu_{1} \in[0,1)$. This and condition (i) imply that

$$
\begin{aligned}
\left(L+J^{-1} P\right) x_{1} & =\mu_{1}\left(N+J^{-1} P\right) x_{1} \\
& \leq \mu_{1}\left(L+J^{-1} P\right) B x_{1} .
\end{aligned}
$$

Applying $\left(L+J^{-1} P\right)^{-1}$ to the above inequality, we obtain $x_{1} \leq \mu_{1} B x_{1}$. Continuing this process, we get

$$
x_{1} \leq \mu_{1}^{n} B^{n} x_{1} .
$$

Let $\Sigma=\left\{y \mid y \geq x_{1}\right\}$. Equation (3.2) yields $\left\{\mu_{1}^{n} B^{n} x_{1} \mid n=1,2, \ldots\right\} \subset \Sigma . x_{1} \in \partial \Omega_{K}$ and $\theta \in \Omega_{K}$ imply $d=d(\theta, \Sigma)>0$. Consequently, we get by (3.2)

$$
\left\|B^{n}\right\| \geq \frac{1}{\left\|x_{1}\right\|}\left\|B^{n} x_{1}\right\| \geq \frac{d}{\mu_{1}^{n}\left\|x_{1}\right\|}, \quad \text { for } n=1,2, \ldots
$$


This shows

$$
r(B)=\lim _{n \rightarrow \infty} \sqrt[n]{\left\|B^{n}\right\|} \geq \frac{1}{\mu_{1}}>1 .
$$

This contradicts the condition (ii). Hence (3.1) is true and we see from Lemma 2.2 that the conclusion is true.

Theorem 3.2 Let $K$ be a normal cone in $X$. If there exist a constant $C>0$ and $u_{1} \in K_{1} \backslash\{\theta\}$, such that

$$
\left(N+J^{-1} P\right) x \geq C\left(L+J^{-1} P\right) x-u_{1}, \quad \text { for all } x \in K
$$

where this partial order is induced by the cone $K_{1}$ in $Y$, then there exists $R_{0}>0$, and for $R>R_{0}$, the fixed point index

$$
\operatorname{ind}_{K}\left([L, N], B_{R} \cap K\right)=\{0\},
$$

where $B_{R}=\left\{x \in X:\|x\|_{X}<R\right\}$.

Proof Setting

$$
W=\left\{x \in K \mid L x-N x=\lambda u_{1} \text {, for each } \lambda \geq 0\right\},
$$

we claim that $W$ is bounded. For $x \in W$, there exists $\lambda \geq 0$ such that $L x-N x=\lambda u_{1}$. This and assumption (3.3) imply that

$$
\left(L+J^{-1} P\right) x=\left(N+J^{-1} P\right) x+\lambda u_{1} \geq\left(N+J^{-1} P\right) x \geq C\left(L+J^{-1} P\right) x-u_{1} .
$$

Operating on both sides of the latter inequality by $\left(L+J^{-1} P\right)^{-1}$, we obtain

$$
x \geq C x-\left(L+J^{-1} P\right)^{-1} u_{1} .
$$

This shows that $x \leq \frac{1}{C-1}\left(L+J^{-1} P\right)^{-1} u_{1}$. In view of the normality of $K$, there exists an $\tilde{N}>0$ such that

$$
\|x\| \leq \frac{\tilde{N}}{C-1}\left\|\left(L+J^{-1} P\right)^{-1} u_{1}\right\|, \quad x \in W .
$$

This shows that $W$ is bounded.

Let $R_{0}=\sup _{x \in W}\|x\|$. For $R>R_{0}$, we have

$$
L x-N x \neq \lambda u_{1}, \quad \forall x \in \partial B_{R} \cap K, \lambda \geq 0 .
$$

Using Lemma 2.3, we infer by (3.4) that the conclusion is true.

Now the following two corollaries are immediate consequences of Theorems 3.1 and 3.2 and the facts that $\left(L+J^{-1} P\right)^{-1}\left(N+J^{-1} P\right)=P+J Q N+L_{1}^{-1}(I-Q) N$ (see [18], Lemma 2) and we have linearity and positivity of the operator $\left(L+J^{-1} P\right)^{-1}$ (see [18], Proposition 1$)$. 
Corollary 3.3 Let $\theta \in \Omega_{K}$ and $L x \neq N x$ for all $x \in \partial \Omega_{K}$. If there exists a positive bounded linear operator $B: X \rightarrow X$ with $r(B) \leq 1$, such that

$$
\left(P+J Q N+L_{1}^{-1}(I-Q) N\right) x \leq B x, \quad \text { for any } x \in \partial \Omega_{K},
$$

where this partial order is induced by the cone $K$ in $X$, then the fixed point index

$$
\operatorname{ind}_{K}\left([L, N], \Omega_{K}\right)=\{1\}
$$

Corollary 3.4 Let $K$ be a normal cone in $X$. If there exist a constant $C>1$ and $u_{0} \in K \backslash\{\theta\}$, such that

$$
\left(P+J Q N+L_{1}^{-1}(I-Q) N\right) x \geq C x-u_{0}, \quad \text { for any } x \in K,
$$

where this partial order is induced by the cone $K$ in $X$, then there exists $R_{0}>0$, and for $R>R_{0}$, the fixed point index

$$
\operatorname{ind}_{K}\left([L, N], B_{R} \cap K\right)=\{0\},
$$

where $B_{R}=\left\{x \in X:\|x\|_{X}<R\right\}$.

Using Corollaries 3.3 and 3.4, we complete this section with a proof of the following important result to be used later.

Theorem 3.5 Let $K$ be a normal cone in $X, u_{0} \in K \backslash\{\theta\}$ and let there be a constant $C>1$. If there exists a positive bounded linear operator $B: X \rightarrow X$ with $r(B) \leq 1$, such that the following hypotheses hold:

(i) $\left(P+J Q N+L_{1}^{-1}(I-Q) N\right) x \geq C x-u_{0}, \forall x \in K$,

(ii) $\left(P+J Q N+L_{1}^{-1}(I-Q) N\right) x \leq B x, \forall x \in \partial B_{r} \cap K$, where $B_{r}=\{x \in X:\|x\|<r\}$, then there exists $x^{*} \in \operatorname{dom} L \cap K \backslash\{\theta\}$ such that $L x^{*}=N x^{*}$.

Proof It follows from Corollary 3.4 and condition (i) that there exists $R>0$ with $R>r$ such that

$$
\operatorname{ind}_{K}\left([L, N], B_{R}\right)=\{0\} .
$$

We assume $L x \neq N x$ on $\partial B_{r} \cap K \cap \operatorname{dom} L$, otherwise the conclusion follows. Using Corollary 3.3 we get from condition (ii)

$$
\operatorname{ind}_{K}\left([L, N], B_{r}\right)=\{1\} .
$$

In view of (3.5), (3.6), and the additivity property, we obtain

$$
\begin{aligned}
\operatorname{ind}_{K}\left([L, N], B_{R} \backslash B_{r}\right) & =\operatorname{ind}_{K}\left([L, N], B_{R}\right)-\operatorname{ind}_{K}\left([L, N], B_{r}\right) \\
& =\{0\}-\{1\}=\{-1\} \\
& \neq\{0\},
\end{aligned}
$$

which completes the proof from the existence property. 


\section{Applications to superlinear periodic boundary value problem}

\subsection{First-order periodic boundary value problem}

We shall apply Theorem 3.5 to obtain positive solutions to the following first-order periodic boundary value problem (PBVP):

$$
\left\{\begin{array}{l}
x^{\prime}(t)=f(t, x(t)), \quad t \in(0,1), \\
x(0)=x(1),
\end{array}\right.
$$

where $f:[0,1] \times \mathbb{R}^{+} \rightarrow \mathbb{R}$ is a continuous function.

Let $X=Y=C[0,1]$ with the usual norm $\|x\|=\max _{t \in[0,1]}|x(t)|$. Define the linear operator $L: \operatorname{dom} L \subset X \rightarrow Y,(L x)(t)=x^{\prime}(t), t \in[0,1]$, where

$$
\operatorname{dom} L=\left\{x \in X: x^{\prime} \in C[0,1], x(0)=x(1)\right\}
$$

and $N: X \rightarrow Y$ with

$$
(N x)(t)=f(t, x(t)), \quad t \in[0,1]
$$

It is easy to check that

$$
\begin{aligned}
& \operatorname{Ker} L=\{x \in \operatorname{dom} L: x(t) \equiv c \text { on }[0,1], c \in \mathbb{R}\}, \\
& \operatorname{Im} L=\left\{y \in Y: \int_{0}^{1} y(s) d s=0\right\}, \\
& \operatorname{dim} \operatorname{Ker} L=\operatorname{codim} \operatorname{Im} L=1,
\end{aligned}
$$

so that $L$ is a Fredholm operator of index zero.

Next, define the projections $P: X \rightarrow X$ by

$$
P x=\int_{0}^{1} x(s) d s
$$

and $Q: Y \rightarrow Y$ by

$$
Q y=\int_{0}^{1} y(s) d s .
$$

Furthermore, we define the isomorphism $J: \operatorname{Im} Q \rightarrow \operatorname{Im} P$ as $J y=\beta y$ with $\beta=1$. We may easy verify that the inverse operator $L_{1}^{-1}: \operatorname{Im} L \rightarrow \operatorname{dom} L \cap \operatorname{Ker} P$ of $\left.L\right|_{\operatorname{dom} L \cap \operatorname{Ker} P}: \operatorname{dom} L \cap$ $\operatorname{Ker} P \rightarrow \operatorname{Im} L$ is $\left(L_{1}^{-1} y\right)(t)=\int_{0}^{1} K(t, s) y(s) d s$, where

$$
K(t, s)= \begin{cases}s+1, & 0 \leq s<t \leq 1 \\ s, & 0 \leq t \leq s \leq 1\end{cases}
$$

For notational convenience, we set $G(t, s)=1+K(t, s)-\int_{0}^{1} K(t, s) d s$. We can verify that

$$
G(t, s)= \begin{cases}\frac{3}{2}-(t-s), & 0 \leq s<t \leq 1 \\ \frac{1}{2}+(s-t), & 0 \leq t \leq s \leq 1\end{cases}
$$

and $\frac{1}{2} \leq G(t, s) \leq \frac{3}{2}, t, s \in[0,1]$. 
Define the cone $K$ in $X$ by

$$
K=\left\{x \in X: x(t) \geq 0, x(t) \geq \frac{1}{3}\|x\|, t \in[0,1]\right\},
$$

then $K$ is a normal cone of $X$ (see [25]).

\section{Lemma 4.1 If}

$\left(\mathrm{H}_{1}\right) f(t, x) \geq-\frac{1}{3} x$, for all $t \in[0,1], x \geq 0$,

then $P+J Q N+L_{1}^{-1}(I-Q) N$ is a positive operator, that is,

$$
\left(P+J Q N+L_{1}^{-1}(I-Q) N\right)(K) \subset K .
$$

Proof It follows from condition $\left(\mathrm{H}_{1}\right)$ that

$$
\begin{aligned}
P x+ & J Q N x+L_{1}^{-1}(I-Q) N x \\
= & \int_{0}^{1} x(s) d s+\int_{0}^{1} f(s, x(s)) d s \\
& +\int_{0}^{1} K(t, s)\left(f(s, x(s))-\int_{0}^{1} f(s, x(s)) d s\right) d s \\
= & \int_{0}^{1} x(s) d s+\int_{0}^{1} G(t, s) f(s, x(s)) d s \\
\geq & \int_{0}^{1}\left(1-\frac{1}{3} G(t, s)\right) x(s) d s \geq 0,
\end{aligned}
$$

for all $x \in K$. Thus $\left(P+J Q N+L_{1}^{-1}(I-Q) N\right)(x) \geq 0$.

Now we are ready to prove

$$
(P+J Q N) x(t)+L_{1}^{-1}(I-Q) N x(t) \geq \frac{1}{3}\left\|(P+J Q N) x+L_{1}^{-1}(I-Q) N x\right\| .
$$

Using condition $\left(\mathrm{H}_{1}\right)$, we obtain

$$
\begin{aligned}
\|(P & +J Q N) x+L_{1}^{-1}(I-Q) N x \| \\
& =\max _{t \in[0,1]}\left[\int_{0}^{1} x(s) d s+\int_{0}^{1} G(t, s) f(s, x(s)) d s\right] \\
& =\max _{t \in[0,1]}\left[\int_{0}^{1}\left(1-\frac{1}{3} G(t, s)\right) x(s) d s+\int_{0}^{1} G(t, s)\left(f(s, x(s))+\frac{1}{3} x(s)\right) d s\right] \\
& \leq \frac{5}{6} \int_{0}^{1} x(s) d s+\frac{3}{2} \int_{0}^{1}\left(f(s, x(s))+\frac{1}{3} x(s)\right) d s .
\end{aligned}
$$

From the last inequality, we have from condition $\left(\mathrm{H}_{1}\right)$

$$
\begin{aligned}
& \min _{t \in[0,1]}\left[(P+J Q N) x(t)+L_{1}^{-1}(I-Q) N x(t)\right] \\
& \quad=\min _{t \in[0,1]}\left[\int_{0}^{1} x(s) d s+\int_{0}^{1} G(t, s) f(s, x(s)) d s\right]
\end{aligned}
$$




$$
\begin{aligned}
& =\min _{t \in[0,1]}\left[\int_{0}^{1}\left(1-\frac{1}{3} G(t, s)\right) x(s) d s+\int_{0}^{1} G(t, s)\left(f(s, x(s))+\frac{1}{3} x(s)\right) d s\right] \\
& \geq \frac{1}{2} \int_{0}^{1} x(s) d s+\frac{1}{2} \int_{0}^{1}\left(f(s, x(s))+\frac{1}{3} x(s)\right) d s \\
& =\frac{1}{3}\left[\frac{3}{2} \int_{0}^{1} x(s) d s+\frac{3}{2} \int_{0}^{1}\left(f(s, x(s))+\frac{1}{3} x(s)\right) d s\right] \\
& \geq \frac{1}{3}\left[\frac{5}{6} \int_{0}^{1} x(s) d s+\frac{3}{2} \int_{0}^{1}\left(f(s, x(s))+\frac{1}{3} x(s)\right) d s\right] \\
& \geq \frac{1}{3}\left\|(P+J Q N) x+L_{1}^{-1}(I-Q) N x\right\| .
\end{aligned}
$$

Therefore, $(P+J Q N) x+L_{1}^{-1}(I-Q) N x \in K$.

We can now state and prove our result on the existence of a positive solution for the PBVP (4.1).

Theorem 4.2 Suppose that condition $\left(\mathrm{H}_{1}\right)$ is satisfied. If

$\left(\mathrm{H}_{2}\right) \liminf _{x \rightarrow+\infty} \min _{t \in[0,1]} \frac{f(t, x)}{x}>4$,

$\left(\mathrm{H}_{3}\right) \lim \sup _{x \rightarrow 0^{+}} \max _{t \in[0,1]} \frac{f(t, x)}{x}<0$,

then the PBVP (4.1) has at least one positive solution.

Proof First, we note that $L$, as defined, is Fredholm of index zero, $L_{1}^{-1}$ is compact by ArzelaAscoli theorem and thus $L-\lambda N$ is A-proper for $\lambda \in[0,1]$ by Lemma 2.1.

Condition $\left(\mathrm{H}_{2}\right)$ guarantees that there exist $\epsilon>0$ and $\tau>0$ such that

$$
f(t, x) \geq(4+\epsilon) x, \quad \text { for all } t \in[0,1], x \geq \tau .
$$

Hence,

$$
f(t, x) \geq(4+\epsilon) x-M, \quad \text { for all } t \in[0,1], x \geq 0,
$$

where $M=\max _{0 \leq t \leq 1,0 \leq x \leq \tau}|f(t, x)-(4+\epsilon) x|+1$.

Set $C=1+\frac{\varepsilon}{6}, u_{0}=\frac{3 M}{2}$. Then $C>1, u_{0} \in K \backslash\{\theta\}$. Using (4.2), we obtain

$$
\begin{aligned}
P x & +J Q N x+L_{1}^{-1}(I-Q) N x \\
& =\int_{0}^{1} x(s) d s+\int_{0}^{1} G(t, s) f(s, x(s)) d s \\
& \geq \int_{0}^{1} x(s) d s+(4+\epsilon) \int_{0}^{1} G(t, s) x(s) d s-M \int_{0}^{1} G(t, s) d s \\
& \geq \int_{0}^{1} x(s) d s+\frac{1}{2}(4+\epsilon) \int_{0}^{1} x(s) d s-\frac{3 M}{2} \\
& =\left(3+\frac{\epsilon}{2}\right) \int_{0}^{1} x(s) d s-\frac{3 M}{2} \\
& \geq\left(3+\frac{\epsilon}{2}\right) \cdot \frac{1}{3}\|x\|-\frac{3 M}{2}
\end{aligned}
$$




$$
\begin{aligned}
& \geq\left(1+\frac{\epsilon}{6}\right) x-\frac{3 M}{2} \\
& =C x-u_{0} .
\end{aligned}
$$

This implies that condition (i) of Theorem 3.5 is satisfied.

It follows from condition $\left(\mathrm{H}_{3}\right)$ that there exist $\sigma \in(0,2)$ and $r \in(0, \tau)$ such that

$$
f(t, x) \leq-\sigma x, \quad \text { for all } t \in[0,1], 0 \leq x \leq r .
$$

Take $B x=\left(1-\frac{\sigma}{2}\right) \int_{0}^{1} x(s) d s$. One can see that $B: X \rightarrow X$ is a positive bounded linear operator. It is clear that $r(B)=1-\frac{\sigma}{2}<1$. Thus, by (4.3), we have

$$
\begin{aligned}
P x & +J Q N x+L_{1}^{-1}(I-Q) N x \\
& =\int_{0}^{1} x(s) d s+\int_{0}^{1} G(t, s) f(s, x(s)) d s \\
& \leq \int_{0}^{1} x(s) d s-\sigma \int_{0}^{1} G(t, s) x(s) d s \\
& \leq \int_{0}^{1} x(s) d s-\frac{\sigma}{2} \int_{0}^{1} x(s) d s \\
& =\left(1-\frac{\sigma}{2}\right) \int_{0}^{1} x(s) d s=B x, \quad \text { for all } x \in K \text { with }\|x\| \leq r .
\end{aligned}
$$

This means that condition (ii) of Theorem 3.5 is verified.

We see that all assumptions of Theorem 3.5 are satisfied. The proof is finished.

\subsection{Second-order periodic boundary value problem}

We will discuss the existence of positive solutions of the second-order periodic boundary value problem (PBVP)

$$
\left\{\begin{array}{l}
-x^{\prime \prime}(t)=f(t, x(t)), \quad t \in(0,1), \\
x(0)=x(1), \quad x^{\prime}(0)=x^{\prime}(1),
\end{array}\right.
$$

where $f:[0,1] \times \mathbb{R}^{+} \rightarrow \mathbb{R}$ is a continuous function.

Since some parts of the proof are in the same line as that of Theorem 4.2, we will outline the proof with the emphasis on the difference.

Let Banach spaces $X, Y$ be as in Section 4.1. In this case, we may define

$$
\operatorname{dom} L=\left\{x \in X: x^{\prime \prime} \in C[0,1], x(0)=x(1), x^{\prime}(0)=x^{\prime}(1)\right\},
$$

and let the linear operator $L: \operatorname{dom} L \subset X \rightarrow Y$ be defined by

$$
(L x)(t)=-x^{\prime \prime}(t), \quad \text { for } x \in \operatorname{dom} L, t \in[0,1] .
$$

Then $L$ is Fredholm of index zero,

$$
\operatorname{Ker} L=\{x \in \operatorname{dom} L: x(t) \equiv c \text { on }[0,1], c \in \mathbb{R}\},
$$


and

$$
\operatorname{Im} L=\left\{y \in Y: \int_{0}^{1} y(s) d s=0\right\} .
$$

Define $N: X \rightarrow Y$ by

$$
(N x)(t)=f(t, x(t)), \quad t \in[0,1] .
$$

Thus it is clear that the PBVP (4.5) is equivalent to the operator equation (1.1).

We use the same projections $P, Q$ as in Section 4.1 and define the isomorphism $J$ : $\operatorname{Im} Q \rightarrow \operatorname{Im} P$ as $J y=\beta y$ with $\beta=1$. It is easy to verify that the inverse operator $L_{1}^{-1}: \operatorname{Im} L \rightarrow$ $\operatorname{dom} L \cap \operatorname{Ker} P$ of $\left.L\right|_{\operatorname{dom} L \cap \operatorname{Ker} P}: \operatorname{dom} L \cap \operatorname{Ker} P \rightarrow \operatorname{Im} L$ is

$$
\left(L_{1}^{-1} y\right)(t)=\int_{0}^{1} \Lambda(t, s) y(s) d s
$$

where

$$
\Lambda(t, s)= \begin{cases}\frac{s}{2}(1-2 t+s), & 0 \leq s<t \leq 1 \\ \frac{1}{2}(1-s)(2 t-s), & 0 \leq t \leq s \leq 1\end{cases}
$$

Set

$$
H(t, s)=\frac{1}{6}+\Lambda(t, s)-\int_{0}^{1} \Lambda(t, s) d s
$$

We can verify that

$$
H(t, s)= \begin{cases}\frac{1}{4}+\frac{s}{2}(1-2 t+s)+\frac{t^{2}}{2}-\frac{t}{2}, & 0 \leq s<t \leq 1 \\ \frac{1}{4}+\frac{1}{2}(1-s)(2 t-s)+\frac{t^{2}}{2}+\frac{t}{2}, & 0 \leq t \leq s \leq 1\end{cases}
$$

and

$$
\frac{1}{8} \leq H(t, s) \leq \frac{1}{4}, \quad t, s \in[0,1] .
$$

Define a normal cone $K$ in $X$ by

$$
K=\left\{x \in X: x(t) \geq 0, x(t) \geq \frac{2}{5}\|x\|, t \in[0,1]\right\} .
$$

\section{Lemma 4.3 If}

$\left(\mathrm{H}_{4}\right) f(t, x) \geq-3 x$, for all $t \in[0,1], x \geq 0$,

then $P+J Q N+L_{1}^{-1}(I-Q) N$ is a positive operator, that is,

$$
\left(P+J Q N+L_{1}^{-1}(I-Q) N\right)(K) \subset K .
$$


Proof For each $x \in K$, by condition $\left(\mathrm{H}_{4}\right)$

$$
\begin{aligned}
(P+J Q N) x+L_{1}^{-1}(I-Q) N x \\
=\int_{0}^{1} x(s) d s+\frac{1}{6} \int_{0}^{1} f(s, x(s)) d s \\
\quad+\int_{0}^{1} \Lambda(t, s)\left(f(s, x(s))-\int_{0}^{1} f(s, x(s)) d s\right) d s \\
=\int_{0}^{1} x(s) d s+\int_{0}^{1} H(t, s) f(s, x(s)) d s \\
\geq \int_{0}^{1}(1-3 H(t, s)) x(s) d s \geq 0 .
\end{aligned}
$$

Thus $\left(P+J Q N+L_{1}^{-1}(I-Q) N\right)(x) \geq 0$.

We now show that

$$
(P+J Q N) x(t)+L_{1}^{-1}(I-Q) N x(t) \geq \frac{2}{5}\left\|(P+J Q N) x+L_{1}^{-1}(I-Q) N x\right\| .
$$

In fact, we get from condition $\left(\mathrm{H}_{4}\right)$

$$
\begin{aligned}
\|( & +J Q N) x+L_{1}^{-1}(I-Q) N x \| \\
& =\max _{t \in[0,1]}\left[\int_{0}^{1} x(s) d s+\int_{0}^{1} H(t, s) f(s, x(s)) d s\right] \\
& =\max _{t \in[0,1]}\left[\int_{0}^{1}(1-3 H(t, s)) x(s) d s+\int_{0}^{1} H(t, s)(f(s, x(s))+3 x(s)) d s\right] \\
& \leq \frac{5}{8} \int_{0}^{1} x(s) d s+\frac{1}{4} \int_{0}^{1}(f(s, x(s))+3 x(s)) d s .
\end{aligned}
$$

By the above inequality, we have from condition $\left(\mathrm{H}_{4}\right)$

$$
\begin{aligned}
& \min _{t \in[0,1]}\left[(P+J Q N) x(t)+L_{1}^{-1}(I-Q) N x(t)\right] \\
& \quad=\min _{t \in[0,1]}\left[\int_{0}^{1} x(s) d s+\int_{0}^{1} H(t, s) f(s, x(s)) d s\right] \\
& =\min _{t \in[0,1]}\left[\int_{0}^{1}(1-3 H(t, s)) x(s) d s+\int_{0}^{1} H(t, s)(f(s, x(s))+3 x(s)) d s\right] \\
& \quad \geq \frac{1}{4} \int_{0}^{1} x(s) d s+\frac{1}{8} \int_{0}^{1}(f(s, x(s))+3 x(s)) d s \\
& =\frac{2}{5}\left[\frac{5}{8} \int_{0}^{1} x(s) d s+\frac{5}{16} \int_{0}^{1}(f(s, x(s))+3 x(s)) d s\right] \\
& \quad \geq \frac{2}{5}\left[\frac{5}{8} \int_{0}^{1} x(s) d s+\frac{1}{4} \int_{0}^{1}(f(s, x(s))+3 x(s)) d s\right] \\
& \quad \geq \frac{2}{5}\left\|(P+J Q N) x+L_{1}^{-1}(I-Q) N x\right\| .
\end{aligned}
$$

Thus $(P+J Q N) x+L_{1}^{-1}(I-Q) N x \in K$. 
Theorem 4.4 Suppose that condition $\left(\mathrm{H}_{4}\right)$ is satisfied. If

$\left(\mathrm{H}_{5}\right) \liminf _{x \rightarrow+\infty} \min _{t \in[0,1]} \frac{f(t, x)}{x}>12$,

$\left(\mathrm{H}_{6}\right) \lim \sup _{x \rightarrow 0^{+}} \max _{t \in[0,1]} \frac{f(t, x)}{x}<0$,

then the PBVP (4.5) has at least one positive solution.

Proof It is again easy to show that $L-\lambda N$ is A-proper for $\lambda \in[0,1]$ by Lemma 2.1. From condition $\left(\mathrm{H}_{5}\right)$, we know that there exist $\epsilon>0$ and $\tau>0$

$$
f(t, x) \geq(12+\epsilon) x, \quad \text { for all } t \in[0,1], x \geq \tau .
$$

Thus

$$
f(t, x) \geq(12+\epsilon) x-M, \quad \text { for all } t \in[0,1], x \geq 0,
$$

where $M=\max _{0 \leq t \leq 1,0 \leq x \leq \tau}|f(t, x)-(12+\epsilon) x|+1$.

From (4.6), we have

$$
\begin{aligned}
P x & +J Q N x+L_{1}^{-1}(I-Q) N x \\
& =\int_{0}^{1} x(s) d s+\int_{0}^{1} H(t, s) f(s, x(s)) d s \\
& \geq \int_{0}^{1} x(s) d s+(12+\epsilon) \int_{0}^{1} H(t, s) x(s) d s-M \int_{0}^{1} H(t, s) d s \\
& \geq \int_{0}^{1} x(s) d s+\frac{1}{8}(12+\epsilon) \int_{0}^{1} x(s) d s-\frac{M}{4} \\
& =\left(\frac{5}{2}+\frac{\epsilon}{8}\right) \int_{0}^{1} x(s) d s-\frac{M}{4} \\
& \geq\left(\frac{5}{2}+\frac{\epsilon}{8}\right) \cdot \frac{2}{5}\|x\|-\frac{M}{4} \\
& \geq\left(1+\frac{\epsilon}{20}\right) x-\frac{M}{4},
\end{aligned}
$$

and condition (i) of Theorem 3.5 is satisfied with $C=1+\frac{\varepsilon}{20}$ and $u_{0}=\frac{M}{4}$.

By condition $\left(\mathrm{H}_{6}\right)$, we can find $\sigma \in(0,8)$ and $r \in(0, \tau)$ such that

$$
f(t, x) \leq-\sigma x, \quad \text { for all } t \in[0,1], 0 \leq x \leq r .
$$

If we take $B x=\left(1-\frac{\sigma}{8}\right) \int_{0}^{1} x(s) d s$, then $B: X \rightarrow X$ is a positive bounded linear operator and $r(B)=1-\frac{\sigma}{8}<1$. Finally, similar to the proof of (4.4), it follows from (4.7) that

$$
P x+J Q N x+L_{1}^{-1}(I-Q) N x \leq B x, \quad \text { for all } x \in K,\|x\| \leq r .
$$

Thus condition (ii) of Theorem 3.5 is satisfied and the proof is complete.

Example 4.5 Let the nonlinearity in (4.5) be

$$
f(t, x)=a(t) x^{\alpha}-k x,
$$


where $\alpha>1, a \in C[0,1]$ is positive 1-periodic function and $k \in(0,3)$. Then (4.5) has at least one positive 1-periodic solution.

Proof We will apply Theorem 4.4 with $f(t, x)=a(t) x^{\alpha}-k x$. Since $k \in(0,3)$, it is easy to see that $\left(\mathrm{H}_{4}\right)$ holds.

One may easily see that $\liminf _{x \rightarrow+\infty} \min _{t \in[0,1]} \frac{f(t, x)}{x}=\liminf _{x \rightarrow+\infty} \min _{t \in[0,1]}\left(a(t) x^{\alpha-1}-k\right)=$ $+\infty>12$, which implies that $\left(\mathrm{H}_{5}\right)$ holds.

On the other hand, we have lim $\sup _{x \rightarrow 0^{+}} \max _{t \in[0,1]} \frac{f(t, x)}{x}=\lim _{\sup _{x \rightarrow 0^{+}}} \max _{t \in[0,1]}\left(a(t) x^{\alpha-1}-\right.$ $k)=-k<0$, which implies that $\left(\mathrm{H}_{6}\right)$ holds. Now we have the desired result.

Remark 4.6 In [27, 28], some existence results for the second-order periodic problem were established by Graef, Kong, Wang and Torres, respectively. Their proofs are based on Krasnosel'skii fixed point theorem in cones for completely continuous operators and the proofs are simpler and more clear than the proof presented in our paper. However, for us it seems difficult to obtain the same results in our paper using the fixed point theorem in cones. The main reason is that our condition $\left(\mathrm{H}_{5}\right)$ is weaker than the classical superlinear condition near $x=+\infty$ (that is, $\liminf _{x \rightarrow+\infty} f(t, x) / x=+\infty$ ), yet being used in [27], Theorem 2.1 and [28], Corollary 4.1. Therefore, in a sense, our results improve and generalize those in $[27,28]$.

Remark 4.7 The following natural question concerning the optimality of conditions $\left(\mathrm{H}_{2}\right)$ and $\left(\mathrm{H}_{5}\right)$ remains open to the authors: 'Find an optimal constant $\lambda^{*}$ such that if $\liminf _{x \rightarrow+\infty} f(t, x) / x>\lambda^{*}$ then Theorems 4.2 and 4.4 remain valid.' In other words, 'Are $\left(\mathrm{H}_{2}\right)$ and $\left(\mathrm{H}_{5}\right)$ also necessary conditions in order to get at least one positive solution in Theorems 4.2 and 4.4 respectively?

Competing interests

The authors declare that they have no competing interests.

Authors' contributions

All authors contributed equally and significantly in writing this paper. All authors read and approved the final manuscript.

\section{Author details}

'School of Mathematics and Physics, Changzhou University, Changzhou, 213164, China. ${ }^{2}$ College of Science, Hohai University, Nanjing, 210098, China. ${ }^{3}$ College of Information Sciences and Technology, Hainan University, Haikou, 570228, China.

\section{Acknowledgements}

The authors express their thanks to the referees and the editor for their valuable comments and suggestions. The research of Feng Wang is supported by the National Natural Science Foundation of China (Grant No. 11501055 and No. 11401166), Natural Science Foundation of the Jiangsu Higher Education Institutions of China (Grant No. 15KJB1 10001). The research of Shengjun Li is supported by the National Natural Science Foundation of China (Grant No. 11461016) and Hainan Natural Science Foundation (Grant No. 113001).

Received: 23 February 2015 Accepted: 24 August 2015 Published online: 01 September 2015

\footnotetext{
References

1. Mawhin, J: Topological Degree Methods in Nonlinear Boundary Value Problems. Regional Conf. in Math., vol. 40. Am. Math. Soc., Providence (1979)

2. Górniewicz, L: Topological approach to differential inclusions. In: Granas, A, Frigon, M (eds.) Topological Methods in Differential Equations and Inclusions. NATO ASI Series C, vol. 472, pp. 129-190. Kluwer Academic, Dordrecht (1995)

3. Cid, JA, Infante, G, Tvrdy, M, Zima, M: A topological approach to periodic oscillations related to the Liebau phenomenon. J. Math. Anal. Appl. 423, 1546-1556 (2015)

4. Fonda, A, Toader, R: Periodic orbits of radially symmetric Keplerian-like systems: a topological degree approach J. Differ. Equ. 244, 3235-3264 (2008)

5. Cui, Y: Computation of topological degree in ordered Banach spaces with lattice structure and applications. Appl. Math. 58, 689-702 (2013)
} 
6. Cui, Y, Sun, J: Fixed point theorems for a class of nonlinear operators in Hilbert spaces with lattice structure and application. Fixed Point Theory Appl. 2013, Article ID 345 (2013)

7. Cui, Y, Wang, F, Zou, Y: Computation for the fixed point index and its applications. Nonlinear Anal. 71, 219-226 (2009)

8. Cui, Y, Zhang, X: Fixed points for discontinuous monotone operators. Fixed Point Theory Appl. 2010, Article ID 926209 (2010)

9. Liu, X, Sun, J: Computation of topological degree of unilaterally asymptotically linear operators and its applications. Nonlinear Anal. 71, 96-106 (2009)

10. Sun, J, Liu, X: Computation for topological degree and its applications. J. Math. Anal. Appl. 202, 785-796 (1996)

11. Sun, J, Liu, X: Computation of topological degree in ordered Banach spaces with lattice structure and its application to superlinear differential equations. J. Math. Anal. Appl. 348, 927-937 (2008)

12. Sun, J, Liu, X: Computation of topological degree for nonlinear operators and applications. Nonlinear Anal. 69 4121-4130 (2008)

13. Gaines, RE, Mawhin, J: Coincidence Degree and Nonlinear Differential Equations. Lecture Notes in Math., vol. 568. Springer, Berlin (1977)

14. Nieto, J: Existence of solutions in a cone for nonlinear alternative problems. Proc. Am. Math. Soc. 94, 433-436 (1985)

15. O'Regan, D, Zima, M: Leggett-Williams norm-type theorems for coincidences. Arch. Math. 87, 233-244 (2006)

16. Santanilla, J: Existence of nonnegative solutions of a semilinear equation at resonance with linear growth. Proc. Am. Math. Soc. 105, 963-971 (1989)

17. Fitzpatrick, PM, Petryshyn, $W V$ : On the nonlinear eigenvalue problem $T(u)=\lambda C(u)$, involving noncompact abstract and differential operators. Boll. Unione Mat. Ital., B 15, 80-107 (1978)

18. Cremins, CT: A fixed-point index and existence theorems for semilinear equations in cones. Nonlinear Anal. 46, 789-806 (2001)

19. Cremins, CT: Existence theorems for semilinear equations in cones. J. Math. Anal. Appl. 265, 447-457 (2002)

20. Chu, J, Wang, F: An order-type existence theorem and applications to periodic problems. Bound. Value Probl. 2013, Article ID 37 (2013)

21. Zhang, F, Wang, F: On existence theorems for semilinear equations and applications. Ann. Pol. Math. 107, 123-131 (2013)

22. Wang, F, Zhang, F: Some new approach to the computation for fixed point index and applications. Bull. Malays. Math. Soc. 36, 491-500 (2013)

23. Amann, H: Fixed point equations and nonlinear eigenvalue problems in ordered Banach spaces. SIAM Rev. 18, 620-709 (1976)

24. Deimling, K: Nonlinear Functional Analysis. Springer, Berlin (1985)

25. Guo, D, Lakshmikantham, V: Nonlinear Problems in Abstract Cones. Academic Press, San Diego (1988)

26. Petryshyn, WV: Using degree theory for densely defined A-proper maps in the solvability of semilinear equations with unbounded and noninvertible linear part. Nonlinear Anal. 4, 259-281 (1980)

27. Graef, JR, Kong, L, Wang, H: A periodic boundary value problem with vanishing Green's function. Appl. Math. Lett. 21, 176-180 (2008)

28. Torres, P: Existence of one-signed periodic solutions of some second-order differential equations via a Krasnosel'skii fixed point theorem. J. Differ. Equ. 190, 643-662 (2003)

\section{Submit your manuscript to a SpringerOpen ${ }^{\circ}$ journal and benefit from:}

- Convenient online submission

Rigorous peer review

- Immediate publication on acceptance

- Open access: articles freely available online

- High visibility within the field

- Retaining the copyright to your article 